\title{
Influence of Thin Water Film on Skid Resistance
}

\author{
Minh-Tan Do, Veronique Cerezo, Yannick Beautru and Malal Kane \\ IFSTTAR (French Institute of Science and Technology for Transport, Development and Networks), Bouguenais 44344, France
}

\begin{abstract}
Most of past researches on the skid resistance/road wetness relationship deal with thick water depths (>1 mm). Questions remain as to the variation of skid resistance with thin water films and the transition between the dry state and the so-called "damp" or "humid" state at which the skid resistance drop can be as high as $30 \%-40 \%$. This paper deals with a theoretical and experimental assessment of the friction-water depth relationship. The main objective is to estimate local water depths trapped between the tire and the road asperities and to define a so-called "critical" water depth which can be used to detect risky situations for road users. Tests are performed in laboratory. It was found that the friction-water depth curves have an inverse-S shape and present an initial constant-friction part before decreasing to a minimum value. A "critical" water depth, defined as the water depth above which the friction coefficient collapses significantly, is determined from observed friction-water depth curves. Influence of test speed and road surface texture on critical water depth is discussed.
\end{abstract}

Key words: Skid resistance, water depth, microtexture.

\section{Introduction}

It is well known that tire/road friction decreases when the road surface is wet. Moore [1] showed that water acts as a lubricant and reduces the fraction of the tire/road contact area where friction forces are generated. Despite this widely accepted explanation, few works have dealt with the relationship between the water depth and the tire/road friction. Based on friction tests using a vehicle equipped with trailer, Veith [2] showed that the friction coefficient is independent of water depth at low speeds (up to 50 $\mathrm{km} / \mathrm{h}$ ) but it is strongly influenced by water depth at high speeds ( $96 \mathrm{~km} / \mathrm{h}$ or greater). It was found that the friction coefficient varies as the logarithm of water depth. Water depths greater than $0.12 \mathrm{~mm}$ were studied.

Models were also published on the calculation of the so-called "hydroplaning speed" $[1,2]$ defined as the speed above which the driver can no more act on his vehicle to control its trajectory. The related situation called "hydroplaning" or aquaplaning occurs on flooded roads.

Corresponding author: Minh-Tan Do, Dr., research field: skid resistance. E-mail: minh-tan.do@ifsttar.fr.
Even if the mentioned works have contributed significantly to the reduction of hydroplaning risk, knowledge is still needed regarding the effect of thin water film on tire/road friction. This situation occurs after rainfalls or during drizzles where the damp aspect of the road surface provides a safety feeling - driving speeds are then as high as those practiced on dry roads. Nevertheless, experimental evidences [3] showed that friction at a "damp" state can already be significantly lower than that at a "dry" state. This drastic drop of friction coefficient explains why accident records are generally high after rainfalls. The tire/road contact loss on damp road surfaces is sometime referred to as "viscoplaning" in order to emphasize the viscous effect of thin water depths.

\section{Research Needs and Methodology}

The single paper dealing with thin water depths is based on works conducted by Kulakowski and Harwood [4]. Using a dedicated laboratory device, they performed friction tests at different water depths and found that the relationship between the friction coefficient and the water depth can be approximated by an exponential function (Fig. 1): 




Fig. 1 Kelationshıp between tıre-pavement triction and water-film thickness [5].

$$
\mu=\Delta \mu \cdot e^{-\beta \cdot h}+\mu_{F}
$$

where,

$\mu$ represents friction coefficient;

$h$ represents water depth;

$\mu_{F}$ represents "final" friction coefficient;

$\Delta \mu$ is the difference between $\mu(0)(\mu$ at $h=0)$ and $\mu_{F}$;

$\beta$ represents parameter of the model.

Actually, Kulakowski and Harwood [4] supposed that the friction coefficient reaches a level-that is $\mu_{\mathrm{F}}$ - at which there is no more variation by increasing water depths. These authors defined a critical water depth $\left(h_{c r i t}\right)$ as the depth at which the dry friction $\mu(0)$ has lost an equivalent of $75 \%$ of $\Delta \mu$ (Fig. 1). The $75 \%$ threshold was chosen arbitrarily. Field tests were conducted at $64 \mathrm{~km} / \mathrm{h}$ to study the influence of the asphalt formulation and the tire on the induced critical water depth. Results indicate that critical water depth lies between $0.025 \mathrm{~mm}$ and $0.23 \mathrm{~mm}$ for different combinations of pavement surfaces and tire types. It is important to notice that the tested pavement surfaces are representative of a wide range of roads and results show that, for each test configuration, very thin water film thickness can decrease significantly the friction coefficient. The wide range of values of critical water depth points out the significant influence of both surface texture and tire.

Research on the effect of thin water depths on tire/road friction is still needed to get a more comprehensive understanding of lubricated tire/road contact. In addition to scientific interests, results of this research can be useful for road authorities looking for a way to inform road users about slip risks under bad weather conditions. Applications can also be developed by car and tire manufacturers to assist drivers unaware of slippery road.

IFSTTAR (French Institute of Science and Technology for Transport, Development and Networks) has initiated a research aiming at developing a model predicting the onset of visco- and hydroplaning from the knowledge of road materials, tire characteristics and tire/road contact conditions (speed, wheel slip, water depth, etc.). The work presented in this paper is part of the Ph.D. carried out by the third author and focused on the viscoplaning aspect. It is composed of three parts:

- In the first part, friction tests at different water depths from dry to flooded states are presented;

- In the second part, the shape of the friction-water depth plot is presented;

- In the third part, definition of a critical water depth is derived in a more physical way than it has been done up to now. Analyses are then conducted to assess the influence of various test conditions on the critical water depth.

\section{Friction Tests}

\subsection{Dynamic Friction Tester Machine}

The DFT (dynamic friction tester) is widely used in North America, Asia and Oceania whereas it is not the case in France-where the first DFT device was acquired in 2009. The machine is composed of a measuring unit (Fig. 2) and a control unit. The measuring unit consists of a horizontal fly wheel and disc which are driven by a motor. Three rubber sliders are attached to the disc by leaf springs. They are pressed on the test surface by the weight of the device and are loaded to $11.8 \mathrm{~N}$ each.

The slider (Fig. 3) is a rubber pad, whose dimensions are $6 \mathrm{~mm} \times 16 \mathrm{~mm} \times 20 \mathrm{~mm}$, bonded to a 


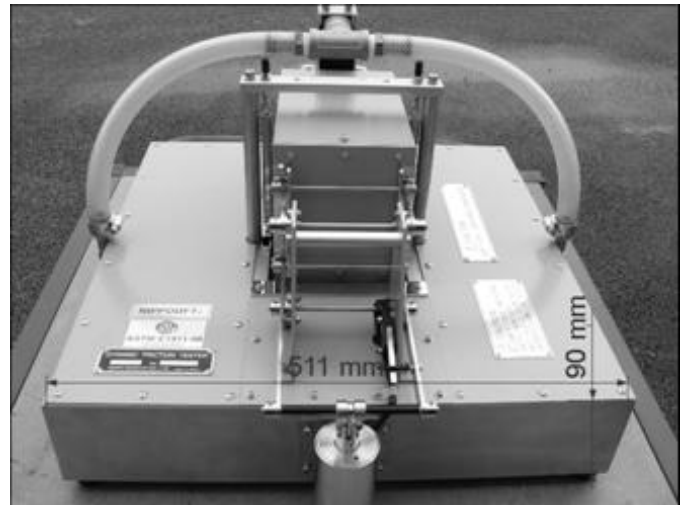

(a)

Fig. 2 DFT (Dynamic friction tester).

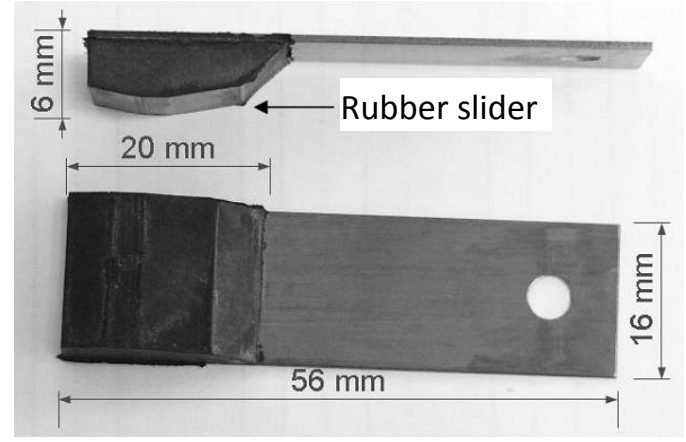

Fig. 3 DFT rubber slider.

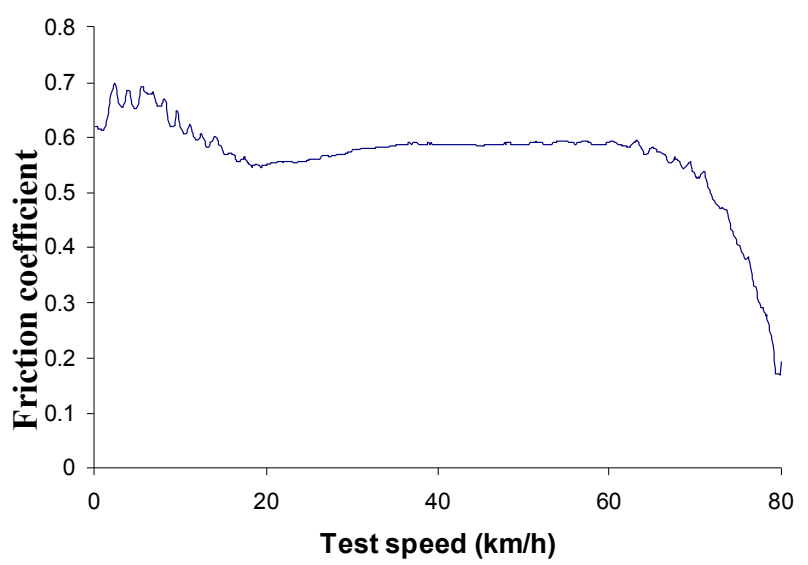

Fig. 4 Typical DFT braking curve.

steel plate. The rubber pad is shaped to provide a contact pressure of $0.15 \mathrm{MPa}$. Full sliding conditions occur in the contact area between the DFT sliders and the test surface. The slider dimensions as well as the contact condition might then simulate tire tread rubber blocks sliding during a locked-wheel braking.

The test procedure is standardized by ASTM (American Society for Testing and Materials) [5]. The test wheel (Fig. 2) is accelerated until it reaches a

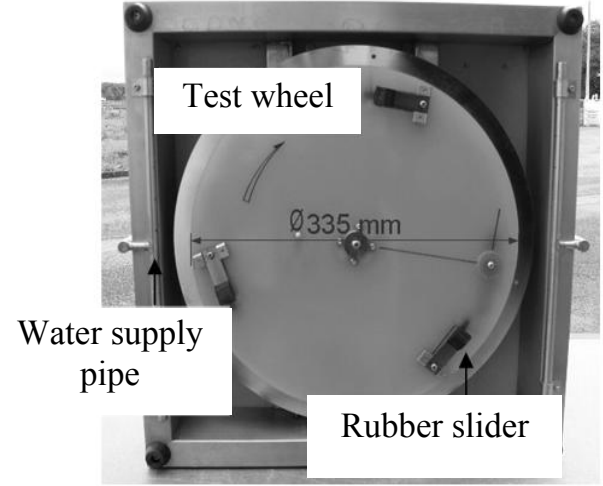

(b)

linear speed of $80 \mathrm{~km} / \mathrm{h}$. Water, provided by a water supply, is projected on the test surface by means of two pipes. The water depth is $1 \mathrm{~mm}$ by the time the test wheel speed reaches $80 \mathrm{~km} / \mathrm{h}$ and the measurement is initiated. The motor is then stopped and the test wheel is dropped. When the rubber sliders are in contact with the test surface, the wheel rotational speed decreases due to the friction generated between the sliders and the surface. Due to the forces on the rubber sliders, displacement occurs in a spring balance. This displacement is then converted into an electrical signal. The speed of rubber sliders is measured from the output of a rotational speed dynamo.

The measurement output is a braking curve from 80 $\mathrm{km} / \mathrm{h}$ to complete stop (Fig. 4). Values of the friction coefficient, typically at 20, 40 and $60 \mathrm{~km} / \mathrm{h}$, are extracted, recorded and displayed on the screen of the control unit.

\subsection{Measurement of Water Depths}

There are different ways to define a water depth, two of which are shown in Fig. 5.

The water depth above asperity summits can be measured by means of devices equipped with needles (usually two conducting electrodes moving vertically). The main drawback of this method is that the measurement is local and cannot represent the mean water depth on the surface unless measurements are done at many locations. Non-contact optical water 


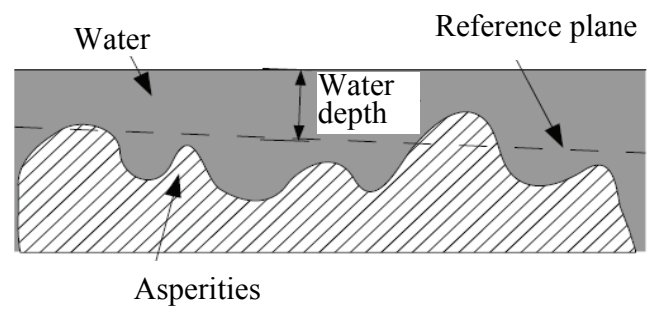

(a)

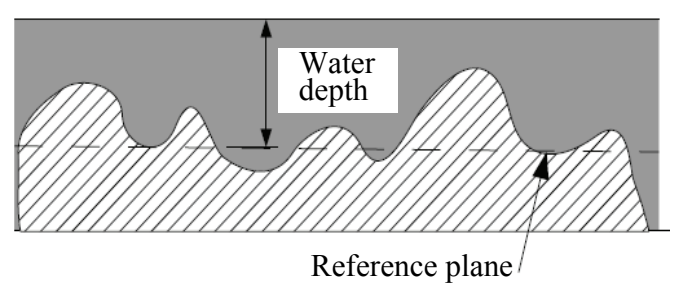

(b)

Fig. 5 Definitions of water depth: (a) above-asperity; (b) centerline-average [2].

depth sensors can be used, too. Unfortunately, devices available at IFSTTAR cannot measure water depths less than $1 \mathrm{~mm}$ - range of interest for this study.

Thereby, a more basic method was chosen to evaluate the wetness of the surface. A spray was used to wet the surface. Before and after each friction measurement, the spray is weighed with an accurate device to know the amount of water sprayed on the test surface (Fig. 6). Dividing the volume of water by the wetted area, an average water depth can be calculated. This method takes into account the fact that water can fill voids in case of a rough surface. The derived water depth corresponds then to the second definition given in Fig. 5.

The calculated water depth is called the "initial equivalent water depth" as it is the thickness of the water film before the friction test is performed. Actually, when the test wheel is in contact with the surface and spins, the water film thickness becomes non-uniform. The determination of actual depths is complex and can be done only by models such as the one developed by Moore [6]. In the present study, the "initial equivalent water depth" is used for further analyses and referred to as, for the sake of simplicity, "water depth".

\subsection{Specimens}

Specimens used in this study are $520 \mathrm{~mm} \times 375$ $\mathrm{mm} \times 30 \mathrm{~mm}$ slabs. Four slabs are produced in laboratory: a VTAC (very thin asphalt concrete) 0/6 (the numbers indicate the size range, in mm, of coarse aggregates); a SCAC (semi-coarse asphalt concrete) $0 / 6$; a sand-asphalt and a mosaic composed of river coarse aggregates. Moreover, to study the effect of surface microtexture, the aggregate mosaic is sandblasted using $590 \mu \mathrm{m}$ corundum particles to simulate a microtextured surface. Actually, the sandblasting roughens the aggregate surface and, by



Fig. 6 Spray weighing.

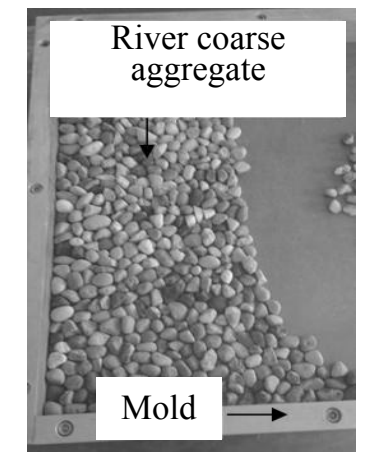

(a)

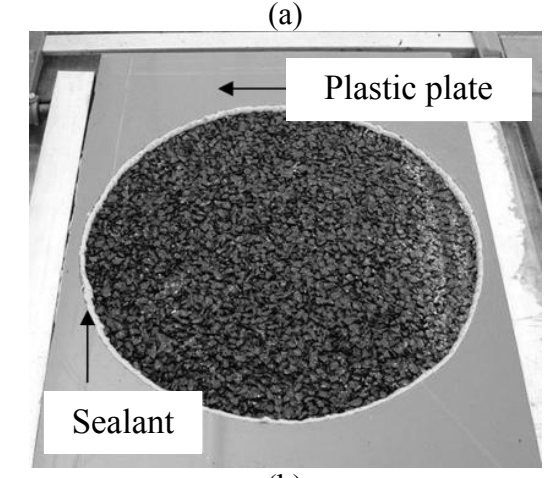

(b)

Fig. 7 (a) Preparation of the mosaic; (b) VTAC specimen prepared for friction tests. 
consequent, modifies only the surface microtexture. VTAC and SCAC are representative of asphalt formulations used for main and secondary roads in France. Asphalt slabs are made by means of IFSTTAR compactor. The mosaic is made by placing coarse aggregates in a mold (Fig. 7a) then covering the bottom with sand and resin.

\subsection{Test Procedure}

The wetted surface is delimited by a plastic plate, in which a circle of $345 \mathrm{~mm}$ of diameter was cut (Fig. 7b), affixed to the specimen slab. The edge of the circle is filled with a sealant to avoid water flowing from the test area. Finally, the slabs are covered, except on their upper face, by a layer of waterproof material in order to prevent the water from flowing out of the sample. The spraying operation can be considered reasonably accurate with the specimens used for the tests. Actually, when $6 \mathrm{~g}$ (average sprayed quantity before each friction measurement) is sprayed on 0.1 $\mathrm{m}^{2}\left(\approx \pi \times\left(\frac{0.345}{2}\right)^{2}\right)$ and, assuming that $10 \%$ of this amount of water is sprayed outside the measurement area, it implies an error of only $0.006 \mathrm{~mm}$ on the estimated water depth.

For each friction test, new sliders are used to ensure that slider wear does not affect results. The test surface is leveled and free of any contamination. The DFT is placed above the slab using visual markers to ensure that it is always placed at the same location. Compared with ASTM (American Standard Test Method) [5], the test procedure is modified to study the influence of water depth on friction coefficient. Actually, the machine is programmed to run tests with no water from the supply unit, water is then added uniquely by the spray. After a first measurement performed on a perfectly dry surface, the following procedure is repeated 12 times:

- Wetting of the slab surface by nine sprayings $(\approx$ $6 \mathrm{~g}$ of water);

- Performing a friction measurement;
- Weighing of the spray.

At the end of this procedure, a test using the standard procedure for the DFT is performed.

\section{Friction-Water Depth Plot}

\subsection{Shape of the Friction-Water Depth Curve}

Example of friction-water depth plots derived from friction tests is shown in Fig. 8. The X-axis represents the water depth. The Y-axis represents the DFT friction coefficient. Friction coefficient obtained by applying the ASTM procedure [5] is also shown.

The observed shape is different from the one found in previous works where an exponential variation of friction with water depth is observed [2, 4]. During the first tests (unpublished) aiming at developing an appropriate test procedure, water was sprayed on the primarily dry surface until it appears wet. This procedure induced actually an exponential variation of friction with water depth. The difference between the Fig. 8 and published results can then be attributed to the water quantity sprayed on the dry test surface to obtain the first wet state. If too much water is sprayed, the transition from "dry" to "wet" can be missed.

The shape of the curve derived from our experiments is similar to that of the well-known Stribeck curve (Fig. 9), although the X-axis is not the same.

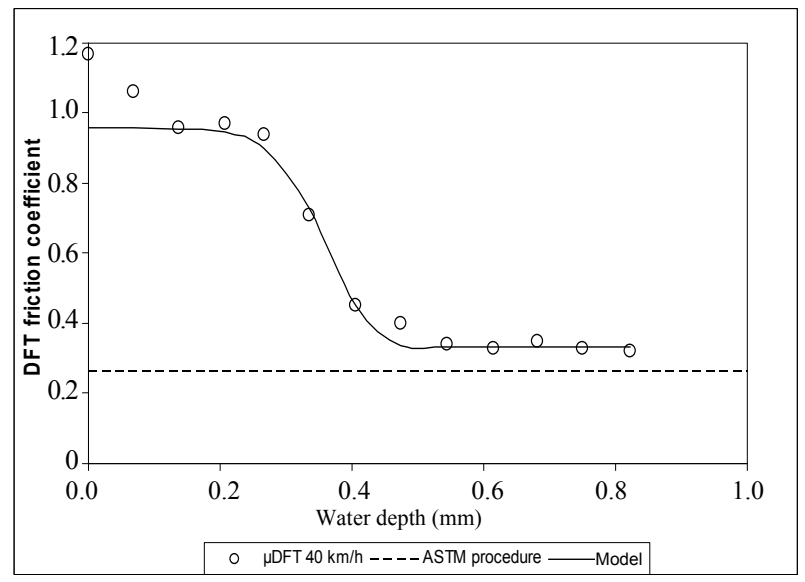

Fig. 8 Example of the variation of friction coefficient versus water depth. 


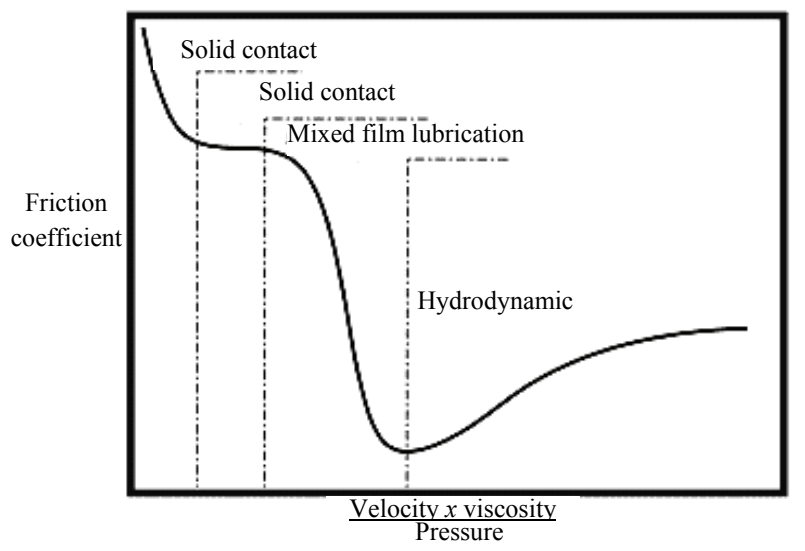

Fig. 9 Stribeck curve [7].

The Stribeck curve relates relative velocity $(V)$, fluid viscosity $(\eta)$ and pressure $(p)$, using the number $\eta V / p$, to friction coefficient. Schipper [8] proved that there exists a relationship between the lubricant depth (h) and $\eta V / p$ in the case of elasto-hydrodynamic lubrication:

$$
h \propto\left(\frac{\eta V}{p}\right)^{0.7} .
$$

Based on Schipper's results, it can reasonably be said that the obtained friction-water depth curve exhibits the same lubrication regimes as those identified in a Stribeck curve (Fig. 9): boundary, mixed and hydrodynamic. It means that the understanding of tire/wet road friction mechanisms, and consequently their modeling, can be enhanced by taking benefit of existing knowledge acquired in tribology. Actually, the graph in Fig. 8 exhibits also a solid-contact phase. This phase is not considered in the following analyses as it occurs only on few surfaces-surfaces covered by a relatively thick binder layer or smooth river coarse aggregates-where water drops do not "stick" to the test surface during the first sprays and then some adhesions might occur.

Fig. 8 shows that the DFT friction coefficient measured by the ASTM procedure represents the lowest friction value that can be expected.

\subsection{Modeling of the Friction-Water Depth Curve}

A model was developed to fit the shape of the friction-water depth curve derived from our experiments:

$$
\mu=\Delta \mu \cdot e^{\left(-\frac{h}{h_{0}}\right)^{\alpha}}+\mu_{F}
$$

where, $h_{0}$ and $\alpha$ represent constants.

The new model is similar to that proposed by Kulakowski and Harwood [4] but can simulate other shapes than the exponential one. Actually, if $\alpha=1$, an exponential variation can be found. For $\alpha \neq 1$, other shapes can be found. The continuous line in Fig. 8 shows how model (3) fits experimental data.

\section{Critical Water Depth}

\subsection{Definition}

The word "critical" is used by road authorities to decide whether warnings must be sent to road users or not. The same word can be used by car manufacturers to activate driver assistance systems. In the context of warning or driver assistance on wet roads, people look for a critical water depth, which can be measured or estimated in real time, above which something must be done. Based on the shape of the friction-water depth curve, it appears that the most critical phase is the transition from boundary to mixed lubrication regimes where friction can drop drastically even if the road surface still displays an apparent "safe" aspect.

Dividing the friction-water depth curve into three parts: boundary, mixed and hydrodynamic lubrication, each part of the friction-water depth curve is linearized as shown in Fig. 10: a horizontal line to represent the stable friction level during the BL phase (boundary lubrication), a sloped line to represent the ML phase (mixed lubrication), and again a horizontal line to represent the HL phase (hydrodynamic lubrication). Actually, friction should increase slightly during the hydrodynamic phase due to drag forces. However, within the experimental setup, no variation curve experienced such a tendency, a horizontal constitutes then a good approximation.

The critical water depth is then defined as the amount of water obtained at the intersection between 


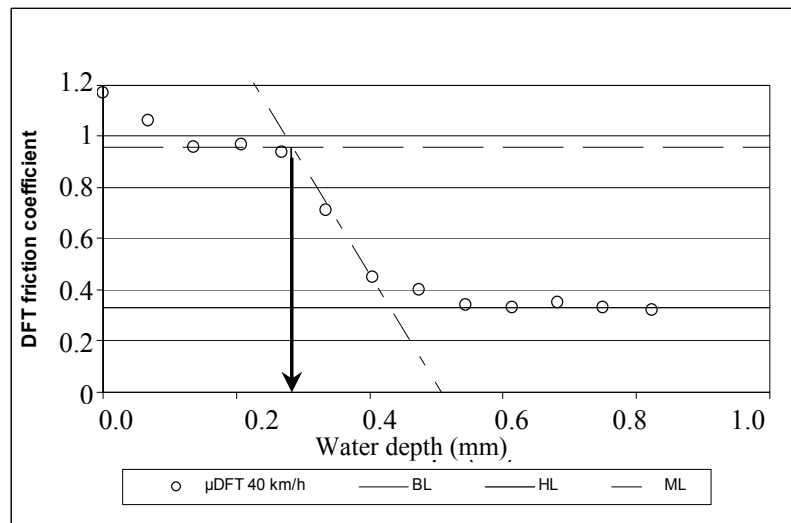

Fig. 10 Linearization of the friction-water depth curve.

the boundary lubrication and mixed lubrication lines (arrow in Fig. 10). From this point, a small additional amount of water is sufficient to degrade significantly the friction coefficient. Within the frame of our experiments, critical water depths lower than $1 \mathrm{~mm}$ were found. Such critical water depths can also determine the onset of viscoplaning as discussed in the introduction.

The remaining of the paper focuses on the critical water depth and highlights how it is affected by factors related to the tire/road contact such as speed or road surface texture.

\subsection{Influence of the Test Speed}

Fig. 11 shows the variation of the critical water depth with the test speed. The VTAC is considered.

Critical water depth tends to decrease with test speed. This tendency is logical since increasing speed leaves less time to evacuate water from the tire/road contact area and, consequently, induces a rapid transition to the mixed lubrication regime. It can be seen that critical water depths are similar at $20 \mathrm{~km} / \mathrm{h}$ and $40 \mathrm{~km} / \mathrm{h}(0.34$ and 0.31 respectively) and higher than that at $60 \mathrm{~km} / \mathrm{h}$ (0.15). It suggests that the effect of speed on the critical water depth is only significant above a certain speed. This confirms observations made by Veith [2].

\subsection{Influence of the Surface Microtexture}

Since the present study deals with thin water films, it is thought that the influence of road surface microtexture is preponderant. Microtexture is conventionally defined as surface irregularities with wavelengths under $0.5 \mathrm{~mm}$ and peak-to-peak amplitudes from $0.001 \mathrm{~mm}$ to $0.5 \mathrm{~mm}$ [9]. It is a function of the surface properties of the aggregate particles contained in the asphalt. It assists in squeezing thin water films in order to provide effective contact between road and tire.

The influence of the surface microtexture can be assessed by comparison of results obtained from the aggregate mosaic before-smooth and after-rough sandblasting. Fig. 12 shows that when the surface is dry, the smooth surface exhibits higher friction value than the sandblasted surface does. This is due to the fact that dry friction depends on available contact area, which is larger on smooth surfaces. However, the

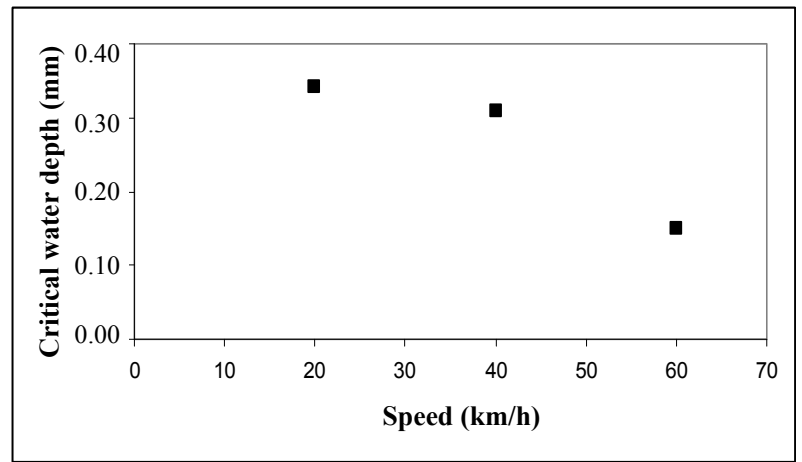

Fig. 11 Variation of critical water depth with speed.

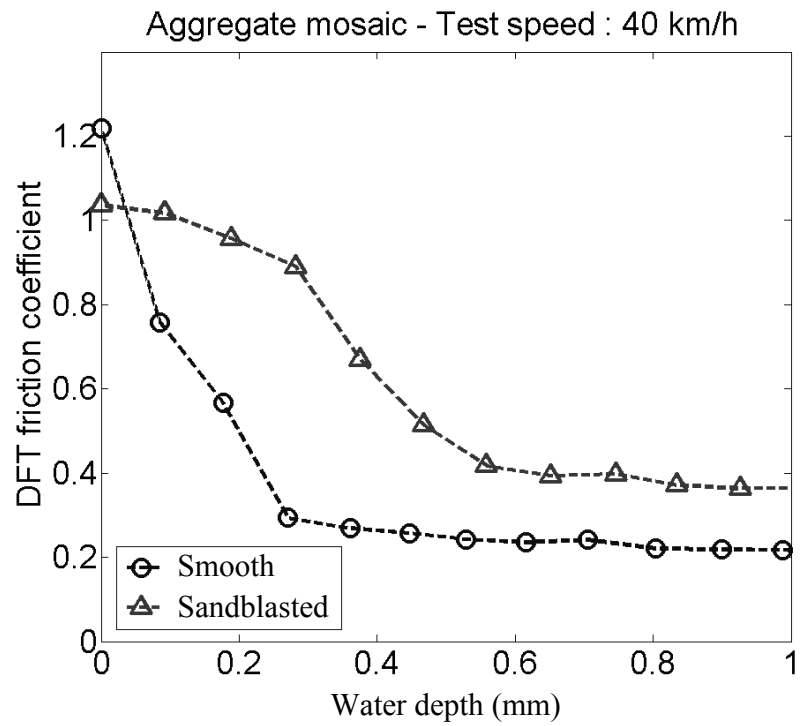

Fig. 12 Influence of surface microtexture on friction coefficient. 
friction coefficient of the mosaic before sandblasting, called "smooth" in Fig. 12, drops rapidly as soon as the surface is wetted. In contrast, the sandblasted mosaic displays a more stable variation of the friction coefficient with the water depth. Also, it can be seen that, even if friction decreases on both surfaces with water depth, the sandblasted curve is always above the smooth curve. These observations corroborate those made by Moore [1] on smooth and roughened spheres.

Fig. 12 clearly highlights the role of the surface microtexture to mitigate the risk of a sudden degradation of wet pavement skid-resistance while maintaining a high friction level. When the surface is wetted, asperities, mainly sharp ones, are needed to squeeze out the water film. Otherwise, as it happened with the smooth mosaic, water, even at very thin thicknesses, can penetrate very quickly in the tire/road contact area and causes contact loss between the tire and the road.

The above analysis is a first attempt to assess the influence of microtexture on the onset of viscoplaning. A more rigorous analysis, using quantitative microtexture descriptors derived from surface profiles, is underway.

\section{Conclusions}

In this paper, study of tire/wet road friction was presented. Focus was made on the effect of thin water films $(<1 \mathrm{~mm})$ which occur after rainfalls or during drizzles. Despite the apparent safety feeling provided by a damp aspect of the road surface, thin water films can alter already significantly the available road friction and reduces contact between the tire and the road.

In the first section, friction tests performed in laboratory were presented. Using a basic method to wet the surface and estimate the average water depth, due to the absence of sensors to measure accurately thicknesses of tenth of millimeter on road surfaces, friction-water depth curves were obtained. Different in shape from previously published curves, the new curves are similar to the well-known Stribeck curve. It was then possible to identify different lubrication regimes occurring at the tire/road interface. Using a simple mathematical function to represent the observed results, a so-called "critical water depth" was derived. It represents the water depth at which transition between the boundary and the mixed lubrication regimes occurs. This new definition, compared with the few ones found in existing literature, is more physical and can be used to determine the onset of viscoplaning.

Further analyses were made to assess the influence of some factors on the critical water depth. It was found that critical water depth decreases with speed, mainly above $60 \mathrm{~km} / \mathrm{h}$ within the frame of our experiments. Observations are logical and can be explained by considering the way by which the water penetrates the tire/road contact area. The influence of road surface microtexture has been clearly demonstrated: friction coefficient on wet microtextured surfaces is maintained at a level comparable to that of a dry surface until a critical water depth is reached, whereas friction coefficient on smooth surfaces drops as soon as the surface is wet. Surface microtexture is then needed to prevent a high degradation of skid resistance. Our results corroborate those from previous studies. Progress has been made because our observations have been made on actual road surfaces whereas those found in the literature are derived from modeled surfaces (spheres, cones, etc.). Efforts, mainly by means of modeling, are still needed to better assess the conditions of viscoplaning onset.

\section{Acknowledgments}

The authors gratefully thank the financial support of the SKIDSAFE (Enhanced Driver Safety due to Improved Skid Resistance) project. Technical support of Mr. Patrick Maisonneuve for laboratory tests is highly appreciated. 


\section{References}

[1] Moore, D. F. 1975. The Friction of Pneumatic Tyres. New York: Elsevier Scientific Publishing Company.

[2] Veith, A. G. 1983. "Tires-Roads-Rainfall-Vehicles: The Traction Connection." In Frictional Interaction of Tire and Pavement, ASTM STP 793, edited by Meyer, W. E., and Reichter, J. West Conshohocken: American Society for Testing and Materials.

[3] Delanne, Y, Do, M. T., Gothié, M., and Delalande, G. 2006. Tyre/Road Friction Potential. ERLPC (Etudes et Recherches des Laboratoires des Ponts et Chaussées). French.

[4] Kulakowski, B. T., and Harwood, D. W. 1990. "Effect of Water-Film Thickness on Tire-Pavement Friction." In Surface Characteristics of Roadways: International Research and Technologies, ASTM STP 1031, edited by
Meyer, W. E., and Reichter, J. West Conshohocken: American Society for Testing and Materials.

[5] American Society for Testing and Materials. 2009. ASTM 2009 Standard Test Method for Measuring Paved Surface Frictional Properties Using the Dynamic Friction Tester. ASTM.

[6] Moore, D. F. 1967. "A Theory of Viscous Hydroplaning." International Journal of Mechanical Sciences 9 (12): 797-810.

[7] Skakoon, J. G. 2009. "There's the Rub." Mechanical Engineering Magazine Online 131 (1): 41-5.

[8] Schipper, D. J. 1988. "Transitions in the Lubrication of Concentrated Contacts." Dissertation, University of Twente, NL.

[9] PIARC (Permanent International Association of Road Congresses). 1987. Report of the Committee on Surface Characteristics. Presented at 18th World Road Congress. 